\title{
USEFUL MARTINGALES FOR STOCHASTIC STORAGE PROCESSES WITH LÉVY-TYPE INPUT
}

\author{
OFFER KELLA, ${ }^{*}$ The Hebrew University of Jerusalem \\ ONNO BOXMA, ${ }^{* *}$ EURANDOM and Eindhoven University of Technology
}

\begin{abstract}
In this paper we generalize the martingale of Kella and Whitt to the setting of Lévy-type processes and show that the (local) martingales obtained are in fact square-integrable martingales which upon dividing by the time index converge to zero almost surely and in $L^{2}$. The reflected Lévy-type process is considered as an example.
\end{abstract}

Keywords: Lévy-type process; Lévy storage system; Kella-Whitt martingale

2010 Mathematics Subject Classification: Primary 60K25; 60K37; 60K30; 60H30

\section{Introduction}

In [9] a certain (local) martingale associated with Lévy processes and its various applications is discussed (see also Section IX.3 of [2] and Section 4.4 of [11]). This has become a standard tool for studying various storage systems with Lévy inputs and other problems associated with Lévy process modeling. In [3] a generalization to a multidimensional (local) martingale associated with Markov additive processes with finite state space Markov modulation is considered, and in [4] a special case of the martingale of [9] for a reflected and a nonreflected Lévy process with no negative jumps and applications to certain hitting times associated with these processes are considered. A generalization to martingales associated with more general functions (than exponential) is given in [13]. The focus is on reflected and nonreflected processes, but the main results seem to hold for the more general structure considered in [9]. There are many papers which apply this and related martingales. As these particular applications are not the scope of this study, we will not attempt to list them here.

The first goal of this paper is to extend the local martingale results of [9] to the case where the driving process is a Lévy-type process. That is, it is a sum of stochastic integrals of some bounded left-continuous, right-limit process with respect to coordinate processes associated with some multidimensional Lévy process. Such processes with an even more general (predictable) integrand are discussed in [1]. The second goal is to extend the original results to show that without any further conditions the resulting local martingales are in fact square-integrable martingales which upon division by the time parameter $t$, converge to 0 almost surely and in $L^{2}$ as $t \rightarrow \infty$. Therefore, certain conditions originally made in [9] to ensure that the local martingales established there are martingales turn out to be unnecessary as Theorem 2 here in particular applies to the special case (Lévy, rather than Lévy type) treated there.

\footnotetext{
Received 20 April 2012; revision received 19 September 2012.

* Postal address: Department of Statistics, The Hebrew University of Jerusalem, Mount Scopus, Jerusalem 91905, Israel. Email address: offer.kella@huji.ac.il

Supported in part by grant 434/09 from the Israel Science Foundation, the Vigevani Chair in Statistics, and visitor grant no. 040.11.257 from The Netherlands Organisation for Scientific Research.

** Postal address: Department of Mathematics and Computer Science, Eindhoven University of Technology, PO Box 513, 5600 MB Eindhoven, The Netherlands. Email address: boxma@win.tue.nl
} 
This paper is organized as follows. In Section 2 we develop the main local martingale. In Section 3 we show that it is in fact a square-integrable martingale and that its rate (defined appropriately) is 0 almost surely and in $L^{2}$. In Section 4 we give a small demonstration of the results with a reflected Lévy-type process and a strong law for Lévy-type processes which is established under some suitable assumptions.

Although following the derivations requires some knowledge, we believe that the final results (in particular Theorem 2 together with Theorem 1 and to a large extent also Corollary 3 and Theorem 3) are quite easy to use by those who are not Lévy process experts nor familiar with the theory of stochastic integration. One particular application that motivated this study is to establish decomposition results for Lévy-driven polling systems (see, e.g. [5] and the references therein), or, more generally, on/off storage systems with Lévy inputs, where the on/off structure could be quite general: during off times the process behaves like a subordinator and during on times it behaves like a (possibly unrelated) reflected Lévy process. These results will be discussed in a separate paper which generalizes [6] and [8], where the results established here are essentially needed and simplify the analysis considerably.

\section{A more general local martingale}

For what follows given a càdlàg (right-continuous, left-limit) function $g: \mathbb{R}_{+} \rightarrow \mathbb{R}$, we define $g(t-)=\lim _{s \uparrow t} g(s)$ and $\Delta g(t)=g(t)-g(t-)$ with the convention that $\Delta g(0)=g(0)$ and if $g$ is VF (finite variation on finite intervals) then $g^{d}(t)=\sum_{0 \leq s \leq t} \Delta g(s)$ and $g^{c}(t)=$ $g(t)-g^{d}(t)$. Also, $\mathbb{R}_{+}=[0, \infty), \mathbb{R}=(-\infty, \infty)$, and a.s. abbreviates almost surely.

Let $X=\left(X_{1}, \ldots, X_{K}\right)$ be a càdlàg $K$-dimensional Lévy process with respect to some standard filtration $\left\{\mathcal{F}_{t} \mid t \geq 0\right\}$ with exponent

$$
\psi(\alpha)=\mathrm{i} c^{\top} \alpha-\frac{\alpha^{\top} \Sigma \alpha}{2}+\int_{\mathbb{R}^{K}}\left(\mathrm{e}^{\mathrm{i} \alpha^{\top} x}-1-\mathrm{i} \alpha^{\top} x \mathbf{1}_{\{\|x\| \leq 1\}}\right) v(\mathrm{~d} x),
$$

where ' $\top$, denotes transposition, $\Sigma$ is positive semidefinite and $\|x\|=\sqrt{x^{\top} x}$. When $X_{1}, \ldots$, $X_{K}$ have no negative jumps, then, for any vector $\alpha \geq 0$, the Laplace-Stieltjes exponent is

$$
\begin{aligned}
\varphi(\alpha) & =\log \mathbb{E} \mathrm{e}^{-\alpha^{\top} X(1)} \\
& =\psi(\mathrm{i} \alpha) \\
& =-c^{\top} \alpha+\frac{\alpha^{\top} \Sigma \alpha}{2}+\int_{\mathbb{R}_{+}^{K}}\left(\mathrm{e}^{-\alpha^{\top} x}-1+\alpha^{\top} x \mathbf{1}_{\{\|x\| \leq 1\}}\right) \nu(\mathrm{d} x) .
\end{aligned}
$$

It is well known that in this case $\varphi(\alpha)$ is finite for each $\alpha \geq 0$, convex (thus continuous) with $\varphi(0)=0$, infinitely differentiable in the interior of $\mathbb{R}_{+}^{K}$, and that, for every $\alpha \geq 0$ for which $\alpha^{\top} X$ is not a subordinator (not nondecreasing), $\varphi(t \alpha) \rightarrow \infty$ as $t \rightarrow \infty$. Furthermore, $\mathbb{E} X_{k}(t)=-t \partial \varphi(0+) / \partial \alpha_{k}$ (finite or $+\infty$, but can never be $-\infty$ ) and, when the first two right derivatives at 0 are finite, then $\operatorname{cov}\left(X_{k}(t), X_{\ell}(t)\right)=t \partial^{2} \varphi(0+) / \partial \alpha_{k} \partial \alpha_{\ell}$.

Lemma 1. Let $I=\left(I_{1}, \ldots, I_{K}\right)$ be a bounded $K$-dimensional adapted càdlàg process. Then

$$
\exp \left[\mathrm{i} \sum_{k=1}^{K} \int_{(0, t]} I_{k}(s-) \mathrm{d} X_{k}(s)-\int_{0}^{t} \psi(I(s)) \mathrm{d} s\right]
$$

is a (complex-valued) martingale. When, in addition, the $X_{k} s$ have no negative jumps and the 
$I_{k} s$ are nonnegative, then

$$
\exp \left[-\sum_{k=1}^{K} \int_{(0, t]} I_{k}(s-) \mathrm{d} X_{k}(s)-\int_{0}^{t} \varphi(I(s)) \mathrm{d} s\right]
$$

is a real-valued martingale.

Proof. The proof follows, for example, by applying a multidimensional generalization of Corollary 5.2.2 and Theorem 5.2.4 of [1, pp. 253-254] to the process

$$
\begin{aligned}
\mathrm{d} Y(t)= & \left(\sum_{k=1}^{K} c_{k} I_{k}(s)-\varphi(I(s))\right) \mathrm{d} t \\
& +\sum_{k=1}^{K}\left(I_{k}(t) \mathrm{d} B_{k}(t)+I_{k}(t-) x \tilde{N}_{k}(\mathrm{~d} t, \mathrm{~d} x)+I_{k}(t-) x N_{k}(\mathrm{~d} t, \mathrm{~d} x)\right),
\end{aligned}
$$

where $Y, B_{k}, N_{k}$, and $\tilde{N}_{k}$ are the notation from [1] with the obvious additional index $k$. Since we will not use this notation in the paper, we only mention them briefly here. Moreover, $Y$ will soon be used for something else, in line with [9] and [3].

Setting $Z(t)=\sum_{k=1}^{K} \int_{(0, t]} I_{k}(s-) \mathrm{d} X_{k}(s)+Y(t)$, the exact same proof from [9] can be employed to prove the following, where $a \wedge b=\min (a, b)$. We recall here that in [9] the driving process was some one-dimensional Lévy process $X$ rather than $\sum_{k=1}^{K} \int_{(0, t]} I_{k}(s-) \mathrm{d} X_{k}(s)$.

Theorem 1. Let $X=\left(X_{1}, \ldots, X_{K}\right)$ be a Lévy process with exponent $\psi$ and, when it has no negative jumps, Laplace-Stieltjes exponent $\varphi$. Let $I=\left(I_{1}, \ldots, I_{K}\right)$ be bounded, càdlàg, and adapted. Assume that $Y$ is càdlàg, VF (a.s.), and adapted. Then

$$
\begin{aligned}
M(t)= & \int_{0}^{t} \psi(I(s)) \mathrm{e}^{\mathrm{i} Z(s)} \mathrm{d} s+\mathrm{e}^{\mathrm{i} Z(0)}-\mathrm{e}^{\mathrm{i} Z(t)}+\mathrm{i} \int_{0}^{t} \mathrm{e}^{\mathrm{i} Z(s)} \mathrm{d} Y^{c}(s) \\
& +\sum_{0<s \leq t} \mathrm{e}^{\mathrm{i} Z(s)}\left(1-\mathrm{e}^{-\mathrm{i} \Delta Y(s)}\right)
\end{aligned}
$$

is a local martingale.

When $Z$ is bounded below, the $X_{k}$ s have no negative jumps and the $I_{k} s$ are nonnegative. Then

$$
\begin{aligned}
M(t)= & \int_{0}^{t} \varphi(I(s)) \mathrm{e}^{-Z(s)} \mathrm{d} s+\mathrm{e}^{-Z(0)}-\mathrm{e}^{-Z(t)}-\int_{0}^{t} \mathrm{e}^{-Z(s)} \mathrm{d} Y^{c}(s) \\
& +\sum_{0<s \leq t} \mathrm{e}^{-Z(s)}\left(1-\mathrm{e}^{\Delta Y(s)}\right)
\end{aligned}
$$

is a local martingale.

We note that in [9] it was assumed that the expected number of jumps of $Y$ on finite intervals is finite in order for the local martingale to be a martingale. It is easy to show with the same proof that the weaker condition

$$
\mathbb{E} \sum_{0<s \leq t}|\Delta Y(s)| \wedge 1<\infty,
$$

is sufficient. For example, if $Y$ is a subordinator (a nondecreasing Lévy process) then it satisfies this condition. Nevertheless, as we will later show that these local martingales are in fact 
square-integrable martingales, even this condition seems unnecessary. We also remark that the condition that $Z$ is bounded below is not really necessary for (1) to be a local martingale, but we will need it later to show that it is a square-integrable martingale with rate 0 , which is the more important result that we are aiming at.

It may seem more general to consider the multidimensional process defined via $Z_{\ell}(t)=$ $\sum_{k=1}^{K} \int_{(0, t]} I_{\ell k}(s-) \mathrm{d} X_{k}(s)+Y_{\ell}$, but we immediately see that the one-dimensional process

$$
\sum_{\ell=1}^{L} \alpha_{\ell} Z_{\ell}(t)=\sum_{k=1}^{K} \int_{(0, t]} \sum_{\ell=1}^{L} \alpha_{\ell} I_{\ell k}(s-) \mathrm{d} X_{k}(s)+\sum_{\ell=1}^{K} \alpha_{\ell} Y_{\ell}(t)
$$

has the same structure, resulting in the following (local) martingales:

$$
\begin{aligned}
M(t)= & \int_{0}^{t} \psi\left(\alpha^{\top} I(s)\right) \mathrm{e}^{\mathrm{i} \alpha^{\top} Z(s)} \mathrm{d} s+\mathrm{e}^{\mathrm{i} \alpha^{\top} Z(0)}-\mathrm{e}^{\mathrm{i} \alpha^{\top} Z(t)} \\
& +\mathrm{i} \sum_{\ell=1}^{L} \alpha_{\ell} \int_{0}^{t} \mathrm{e}^{\mathrm{i} \alpha^{\top} Z(s)} \mathrm{d} Y_{\ell}^{c}(s)+\sum_{0<s \leq t} \mathrm{e}^{\mathrm{i} \alpha^{\top} Z(s)}\left(1-\mathrm{e}^{-\mathrm{i} \alpha^{\top} \Delta Y(s)}\right)
\end{aligned}
$$

and

$$
\begin{aligned}
M(t)= & \int_{0}^{t} \varphi\left(\alpha^{\top} I(s)\right) \mathrm{e}^{-\alpha^{\top} Z(s)} \mathrm{d} s+\mathrm{e}^{-\alpha^{\top} Z(0)}-\mathrm{e}^{-\alpha^{\top} Z(t)} \\
& -\sum_{\ell=1}^{L} \alpha_{\ell} \int_{0}^{t} \mathrm{e}^{-\alpha^{\top} Z(s)} \mathrm{d} Y_{\ell}^{c}(s)+\sum_{0<s \leq t} \mathrm{e}^{-\alpha^{\top} Z(s)}\left(1-\mathrm{e}^{\alpha^{\top} \Delta Y(s)}\right) .
\end{aligned}
$$

Here $I$ is an $(L \times K)$-matrix-valued function.

We note that, when $J$ is a (right-continuous) continuous-time Markov chain with states $1, \ldots, K$, then, with $I_{k}(t)=\mathbf{1}_{\{J(t)=k\}}, \sum_{k=1}^{K} \int_{(0, t]} I_{k}(s-) \mathrm{d} X_{k}(s)$ is a Markov additive process. Adding additional jumps at state change epochs can be modeled by the process $Y$, which is obviously VF. For the case where $Y$ is continuous, this kind of process and associated martingales were considered in [3]. The one-dimensional martingales considered here are not the same as the multidimensional ones considered there. However, the sum of the components of the latter does agree with the former.

We conclude this section with the following observation. Assume that $J$ is a càdlàg adapted process taking values in some finite set $1, \ldots, K$ (not necessarily Markovian). Let $I_{k}(t)=$ $\alpha_{k} \mathbf{1}_{\{J(t)=k\}}$. Then

$$
\psi(I(t))=\sum_{k=1}^{K} \psi_{k}\left(\alpha_{k}\right) \mathbf{1}_{\{J(t)=k\}},
$$

where $\psi_{k}\left(\alpha_{k}\right)=\psi\left(0, \ldots, 0, \alpha_{k}, 0, \ldots, 0\right)$ with $\alpha_{k}$ in the $k$ th coordinate, is defined in the previous remark (and similarly with $\varphi$ when there are no negative jumps). Thus, in this case

$$
\int_{0}^{t} \psi(I(s)) \mathrm{e}^{\mathrm{i} Z(s)} \mathrm{d} s=\sum_{k=1}^{K} \psi_{k}\left(\alpha_{k}\right) \int_{0}^{t} \mathrm{e}^{\mathrm{i} Z(s)} \mathbf{1}_{\{J(s)=k\}} \mathrm{d} s .
$$

If, in addition, we replace $Y$ by $\beta Y$ for some $\beta \geq 0$ and define $\tilde{X}_{k}(t)=\int_{(0, t]} \mathbf{1}_{\{J(s)=k\}} \mathrm{d} X_{k}(s)$, then

$$
Z(t)=\alpha^{\top} \tilde{X}(t)+\beta Y(t)
$$


and the (local) martingale becomes

$$
\begin{aligned}
M(t)= & \sum_{k=1}^{K} \psi_{k}\left(\alpha_{k}\right) \int_{0}^{t} \mathrm{e}^{\mathrm{i} Z(s)} \mathbf{1}_{\{J(s)=k\}} \mathrm{d} s+\mathrm{e}^{\mathrm{i} Z(0)}-\mathrm{e}^{\mathrm{i} Z(t)} \\
& +\mathrm{i} \beta \int_{0}^{t} \mathrm{e}^{\mathrm{i} Z(s)} \mathrm{d} Y^{c}(s)+\sum_{0<s \leq t} \mathrm{e}^{\mathrm{i} Z(s)}\left(1-\mathrm{e}^{-\mathrm{i} \beta \Delta Y(s)}\right),
\end{aligned}
$$

and, similarly,

$$
\begin{aligned}
M(t)= & \sum_{k=1}^{K} \varphi_{k}\left(\alpha_{k}\right) \int_{0}^{t} \mathrm{e}^{-Z(s)} \mathbf{1}_{\{J(s)=k\}} \mathrm{d} s+\mathrm{e}^{-Z(0)}-\mathrm{e}^{-Z(t)} \\
& -\beta \int_{0}^{t} \mathrm{e}^{-Z(s)} \mathrm{d} Y^{c}(s)+\sum_{0<s \leq t} \mathrm{e}^{-Z(s)}\left(1-\mathrm{e}^{\beta \Delta Y(s)}\right)
\end{aligned}
$$

when there are no negative jumps.

It seems that the joint structure of $X$ is not important here. This is partly true in the sense that the evolution of the Lévy part of the process during times when $J$ is at a given state is that of a one-dimensional Lévy process. However, both $J$ and $Y$ may also depend on the joint structure.

\section{3. $M$ is a square-integrable martingale with $M(t) / t \rightarrow 0$ a.s. and in $L^{2}$}

In this section we will show that $M$ is a square-integrable martingale with $M(t) / t \rightarrow 0$ a.s. and in $L^{2}$ as $t \rightarrow \infty$. This is something that was overlooked in [9]. To keep the discussion shorter, we will restrict it to the case of (1) where $Z$ is nonnegative, the $X_{k}$ have no negative jumps, and the $I_{k}$ are nonnegative. The proofs for the general (complex-valued) case are basically identical (but see Remark 1). Assuming the seemingly more general condition that $Z$ is bounded below rather than nonnegative is of no consequence to the proofs. We begin with the following.

Lemma 2. Let $X$ be a semimartingale, and let $f \in \mathcal{C}^{2}$ (twice continuously differentiable). Denote by $[\cdot, \cdot]$ the quadratic variation process associated with a semimartingale. Then $f(X)$ is also a semimartingale with the following quadratic variation:

$$
[f(X), f(X)](t)=\int_{0}^{t}\left(f^{\prime}(X(s))\right)^{2} \mathrm{~d}[X, X]^{c}(s)+\sum_{0 \leq s \leq t}(\Delta f(X(s)))^{2} .
$$

Proof. Although this should have been a standard result in a book (such as [14]) we did not find a direct reference. For its proof, we apply the extended Itô lemma (see Theorem 32 of [14, p. 78]) to conclude that

$$
f(X(t))=f(X(0))+\int_{(0, t]} f^{\prime}(X(s-)) \mathrm{d} X(s)+\text { continuous VF part }+ \text { discrete VF part. }
$$

As in the displayed equation following the definition of $[X, X]^{c}$ on page 70 of [14], we have

$$
[f(X), f(X)](t)=[f(X), f(X)]^{c}(t)+\sum_{0 \leq s \leq t}(\Delta f(X(s)))^{2} .
$$


Finally, we note that the only term that can contribute to the continuous part of the quadratic variation associated with $f(X)$ is the stochastic integral part. Thus, with the notation $f\left(X_{-}\right) \cdot X(t)=\int_{(0, t]} f(X(s-)) \mathrm{d} X(s)$ we now have, via Theorem 29 of [14, p. 75],

$[f(X), f(X)]^{c}=\left[f\left(X_{-}\right) \cdot X, f\left(X_{-}\right) \cdot X\right]^{c}=\left(\left(f^{\prime}\left(X_{-}\right)\right)^{2} \cdot[X, X]\right)^{c}=\left(f^{\prime}\left(X_{-}\right)\right)^{2} \cdot[X, X]^{c}$.

This completes the proof.

Corollary 1. Assume that $X$ is a semimartingale, $Y$ is VF and adapted, and $Z=X+Y$. Then

$$
\left[\mathrm{e}^{-Z}, \mathrm{e}^{-Z}\right](t)=\int_{0}^{t} \mathrm{e}^{-2 Z(s)} \mathrm{d}[X, X]^{c}(s)+\sum_{0 \leq s \leq t} \mathrm{e}^{-2 Z(s-)}\left(1-\mathrm{e}^{-\Delta Z(s)}\right)^{2} .
$$

Proof. The proof follows from $[Z, Z]^{c}=[X, X]^{c}$ (as $Y$ is VF), substitution, and some obvious manipulations.

Remark 1. Given the above, it is now an easy exercise to show that in fact, for $X$ a semimartingale and $f, g \in \mathcal{C}^{2}$, we have

$$
[f(X), g(X)](t)=\int_{0}^{t} f^{\prime}(X(s)) g^{\prime}(X(s)) \mathrm{d}[X, X]^{c}(s)+\sum_{0 \leq s \leq t} \Delta f(X(s)) \Delta g(X(s)),
$$

and to conclude from this that, under the assumptions of Corollary 1 ,

$$
\left[\mathrm{e}^{\mathrm{i} Z}, \mathrm{e}^{\mathrm{i} Z}\right](t)=\int_{0}^{t} \mathrm{e}^{\mathrm{i} 2 Z(s)} \mathrm{d}[X, X]^{c}(s)+\sum_{0 \leq s \leq t} \mathrm{e}^{\mathrm{i} 2 Z(s-)}\left(1-\mathrm{e}^{\mathrm{i} \Delta X(s)}\right)^{2}
$$

by treating the real and imaginary parts separately. This is needed for the general case which, as mentioned, is omitted from the discussion here.

Recall $M$ from (1) and that we are assuming that $X$ has no negative jumps and $I$ is nonnegative.

\section{Corollary 2. It holds that}

$$
[M, M](t)=\int_{0}^{t} \mathrm{e}^{-2 Z(s)} \mathrm{d}[\tilde{X}, \tilde{X}]^{c}(s)+\sum_{0<s \leq t} \mathrm{e}^{-2 Z(s-)}\left(1-\mathrm{e}^{-\Delta \tilde{X}(s)}\right)^{2} .
$$

Proof. The only part of $M$ that can contribute to the quadratic variation is

$$
\sum_{0<s \leq t} \mathrm{e}^{-Z(s)}\left(1-\mathrm{e}^{\Delta Y(s)}\right)+\mathrm{e}^{-Z(0)}-\mathrm{e}^{-Z(t)},
$$

as the rest are continuous and VF. Clearly, only $\mathrm{e}^{-Z(t)}$ contributes to the continuous part of this quadratic variation and, from Corollary 1 , is given by $\int_{0}^{t} \mathrm{e}^{-2 Z(s)} \mathrm{d}[\tilde{X}, \tilde{X}]^{c}(s)$. Since $\mathrm{e}^{-Z(0)}-$ $\mathrm{e}^{-Z(s)}=0$ for $s=0$, the 'jump' at 0 is excluded. Now, as $Z(s)=Z(s-)+\Delta \tilde{X}(s)+\Delta Y(s)$,

$$
\begin{aligned}
& \Delta\left(\sum_{0<s \leq t} \mathrm{e}^{-Z(s)}\left(1-\mathrm{e}^{\Delta Y}\right)+\mathrm{e}^{-Z(0)}-\mathrm{e}^{-Z(t)}\right)(t) \\
& \quad=\mathrm{e}^{-Z(t)}\left(1-\mathrm{e}^{\Delta Y(t)}\right)+\mathrm{e}^{-Z(t-)}-\mathrm{e}^{-Z(t)} \\
& \quad=\mathrm{e}^{-Z(t-)}\left(\mathrm{e}^{-\Delta Y(t)}-1\right) \mathrm{e}^{-\Delta \tilde{X}(t)}+\mathrm{e}^{-Z(t-)}\left(1-\mathrm{e}^{-\Delta Y(t)} \mathrm{e}^{-\Delta \tilde{X}(t)}\right) \\
& =-\mathrm{e}^{-Z(t-)}\left(1-\mathrm{e}^{-\Delta \tilde{X}(t)}\right) .
\end{aligned}
$$


As the discrete part of the quadratic variation is just the sum of squares of these jumps, we are done.

\section{Lemma 3. It holds that}

$$
[M, M](t)=\int_{0}^{t} \mathrm{e}^{-2 Z(s)} A(s) \mathrm{d} s+\tilde{M}(t),
$$

where

$$
A(s)=\varphi(2 I(s))-2 \varphi(I(s))
$$

is nonnegative and bounded, and $\tilde{M}$ is a martingale having bounded jumps.

Proof. Recalling that $\tilde{X}(t)=\sum_{k=1}^{K} \int_{(0, t]} I_{k}(s-) \mathrm{d} X_{k}(s)$, we have, from Theorem 29 of [14, p. 75],

$$
[\tilde{X}, \tilde{X}]=\sum_{k=1}^{K} \sum_{\ell=1}^{K}\left[I_{k} \cdot X_{k}, I_{\ell} \cdot X_{\ell}\right]=\sum_{k=1}^{K} \sum_{\ell=1}^{K} I_{k} I_{\ell} \cdot\left[X_{k}, X_{\ell}\right]
$$

and, thus,

$$
[\tilde{X}, \tilde{X}]^{c}=\sum_{k=1}^{K} \sum_{\ell=1}^{K} I_{k} I_{\ell} \cdot\left[X_{k}, X_{\ell}\right]^{c} .
$$

Now, since we can write $X=B+C$, where $B$ is a Brownian motion and $C$ is a quadratic pure jump Lévy process (see, e.g. top of page 71 of [14]), then $\left[X_{k}, X_{\ell}\right]^{c}(t)=\left[B_{k}, B_{\ell}\right](t)=\sigma_{k \ell} t$, which implies that

$$
[\tilde{X}, \tilde{X}]^{c}(t)=\int_{0}^{\top} I(s)^{\top} \Sigma I(s) \mathrm{d} s=\int_{0}^{t}\left[\frac{\left(2 I(s)^{\top}\right) \Sigma(2 I(s))}{2}-2 \frac{I(s)^{\top} \Sigma I(s)}{2}\right] \mathrm{d} s .
$$

Next, from $\Delta \tilde{X}(s)=\sum_{k=1}^{K} I(s-) \Delta X_{k}(s)$, we observe that

$$
\sum_{0<s \leq t} \mathrm{e}^{-2 Z(s-)}\left(1-\mathrm{e}^{-\Delta \tilde{X}(s)}\right)=\int_{(0, t] \times(0, \infty)^{K}} \mathrm{e}^{-2 Z(s-)}\left(1-\mathrm{e}^{-I^{\top}(s-) x}\right) N(\mathrm{~d} s, \mathrm{~d} x),
$$

where $N$ is the usual Poisson random measure with intensity measure $\mathrm{d} s \otimes v(\mathrm{~d} x)$ associated with the jumps of $X$. Therefore, with $\tilde{N}(\mathrm{~d} s, \mathrm{~d} x)=N(\mathrm{~d} s, \mathrm{~d} x)-\mathrm{d} s \otimes v(\mathrm{~d} x)$, recalling that $\int_{\mathbb{R}^{K}}\left(\|x\|^{2} \wedge 1\right) v(\mathrm{~d} x)<\infty$ and noting that $\mathrm{e}^{-Z(s-)}\left(1-\mathrm{e}^{-I(s-) x}\right) \leq(B\|x\|) \wedge 1$, where $B$ is an upper bound for $\|I(t)\|$ and, thus, $\int_{0}^{t} \int_{\mathbb{R}^{K}} \mathbb{E}^{-2 Z(s-)}\left(1-\mathrm{e}^{-I(s-) x}\right)^{2} v(\mathrm{~d} x) \mathrm{d} s<\infty$, it follows (see, e.g. Proposition 4.10 of [15]) that

$$
\tilde{M}(t)=\int_{(0, t] \times(0, \infty)^{K}} \mathrm{e}^{-2 Z(s-)}\left(1-\mathrm{e}^{-I^{\top}(s-) x}\right)^{2} \tilde{N}(\mathrm{~d} s, \mathrm{~d} x)
$$

is a martingale, necessarily having bounded jumps, and so

$$
\sum_{0<s \leq t} \mathrm{e}^{-2 Z(s-)}\left(1-\mathrm{e}^{-\Delta \tilde{X}(s)}\right)^{2}=\int_{0}^{t} \int_{(0, \infty)^{K}} \mathrm{e}^{-2 Z(s-)}\left(1-\mathrm{e}^{-I^{\top}(s-) x}\right)^{2} v(\mathrm{~d} x) \mathrm{d} s+\tilde{M}(t)
$$

Finally, we observe that, for any $a, x \in \mathbb{R}_{+}^{K}$,

$$
\left(1-\mathrm{e}^{-a^{\top} x}\right)^{2}=\left(\mathrm{e}^{-(2 a)^{\top} x}-1+(2 a)^{\top} x \mathbf{1}_{\{\|x\| \leq 1\}}\right)-2\left(\mathrm{e}^{-a^{\top} x}-1+a^{\top} x \mathbf{1}_{\{\|x\| \leq 1\}}\right),
$$

and upon replacing $a$ by $I(s-)$ and integrating with respect to $v(\mathrm{~d} x)$, then together with (2) and (3), the result is obtained. 
Theorem 2. $M$ is a square-integrable martingale with $M(t) / t \rightarrow 0$ as $t \rightarrow \infty$ a.s. and in $L^{2}$.

Proof. Since $I$ is bounded and $\varphi$ is continuous, then so is $\varphi(I)$. Therefore, there exists a constant $C$ such that $\varphi(2 I(s))-2 \varphi(I(s)) \leq C$ and, thus, $\mathrm{e}^{-2 Z(s)}(\varphi(2 I(s))-2 \varphi(I(s))) \leq C$ also. As $\tilde{M}$ is a zero-mean martingale, Lemma 3 implies that

$$
\mathbb{E}[M, M](t)=\int_{0}^{t} \mathrm{e}^{-2 Z(s)}(\varphi(2 I(s))-2 \varphi(I(s))) \mathrm{d} s \leq C t<\infty,
$$

and, thus, by Corollary 3 of [14, p. 73], $M$ is a square-integrable martingale with $\mathbb{E} M^{2}(t)=$ $\mathbb{E}[M, M](t)$. Similarly, as $\int_{(0, t]}(1+s)^{-2} \mathrm{~d} \tilde{M}(s)$ is a zero-mean martingale, then

$$
\mathbb{E} \int_{0}^{t}(1+s)^{-2} \mathrm{~d}[M, M](s) \leq C \int_{0}^{t}(1+s)^{-2} \mathrm{~d} s=C\left(1-\frac{1}{1+t}\right) \leq C .
$$

Letting $t \rightarrow \infty$ and applying monotone convergence on the left-hand side (again with Corollary 3 of $\left[14\right.$, p. 73]) implies that $\int_{0}^{t}(1+s)^{-1} \mathrm{~d} M(s)$ is a square-integrable martingale with second moment given by the left-hand side of (4), that $\int_{0}^{\infty}(1+s)^{-1} \mathrm{~d} M(s)$ converges a.s., and, thus, Exercise 14 of [14, p. 95] implies that $M(t) /(1+t) \rightarrow 0$, and, hence, also $M(t) / t \rightarrow 0$ a.s. $L^{2}$ convergence is due to $\mathbb{E}(M(t) / t)^{2}=\mathbb{E}[M, M](t) / t^{2} \leq C / t$.

\section{A consequence for the reflected Lévy-type process}

Reflected processes are widely used as models for various storage processes. With

$$
L(t)=-\inf _{0 \leq s \leq t}(Y(0)+\tilde{X}(s))^{-},
$$

it is well known that $Z(t)=\tilde{X}(t)+L(t)=0$ at any point of (right) increase of $L$ (see, e.g. [7]). In the case where $X$ has no negative jumps $L$ is continuous. Thus, in the general case $M$ becomes

$$
\begin{aligned}
M(t) & =\int_{0}^{t} \psi(I(s)) \mathrm{e}^{\mathrm{i} Z(s)} \mathrm{d} s+\mathrm{e}^{\mathrm{i} Z(0)}-\mathrm{e}^{\mathrm{i} Z(t)}+\mathrm{i} L^{c}(t)+\sum_{0<s \leq t}\left(1-\mathrm{e}^{-\mathrm{i} \Delta L(s)}\right) \\
& =\int_{0}^{t} \psi(I(s)) \mathrm{e}^{\mathrm{i} Z(s)} \mathrm{d} s+\mathrm{e}^{\mathrm{i} Z(0)}-\mathrm{e}^{\mathrm{i} Z(t)}+\mathrm{i} L(t)-\sum_{0<s \leq t}\left(\mathrm{e}^{-\mathrm{i} \Delta L(s)}-1+\mathrm{i} \Delta L(s)\right),
\end{aligned}
$$

and, when $X_{k}$ have no negative jumps and the $I_{k}$ are nonnegative, then $\Delta L(s)=0$ and

$$
M(t)=\int_{0}^{t} \varphi(I(s)) \mathrm{e}^{-Z(s)} \mathrm{d} s+\mathrm{e}^{-Z(0)}-\mathrm{e}^{-Z(t)}-L(t) .
$$

By Theorem 2 we therefore have for this case

$$
\frac{1}{t} \int_{0}^{t} \varphi(I(s)) \mathrm{e}^{-Z(s)} \mathrm{d} s-\frac{1}{t} L(t) \rightarrow 0
$$

a.s. and in $L^{2}$. 
Also, we recall from the arguments of Theorem 1 of [10] that (pathwise) $\tilde{X}(t) / t \rightarrow \xi$ if and only if

$$
\left(\frac{Z(t)}{t}, \frac{L(t)}{t}\right) \rightarrow\left(\xi^{+},-\xi^{-}\right),
$$

where $a^{+}=\max (a, 0)$ and $a^{-}=\min (a, 0)$. This is true for any càdlàg $\tilde{X}$, not necessarily having the special structure we consider here. Thus, when $X_{k}$ have no negative jumps and the $I_{k}$ are nonnegative, it now follows that

$$
\frac{1}{t} \int_{0}^{t} \varphi(I(s)) \mathrm{e}^{-Z(s)} \mathrm{d} s \rightarrow-\xi^{-} .
$$

To figure out what $\xi$ is in this case, we use the following result which is related to Theorem 2 and holds regardless of whether there are negative jumps or not.

Lemma 4. Let $X$ be a one-dimensional Lévy process with Lévy measure v satisfying

$$
\int_{|x|>1}|x| v(\mathrm{~d} x)<\infty
$$

(equivalently, $\mathbb{E}|X(1)|<\infty)$. Then, for any bounded càdlàg adapted process $A$,

$$
\frac{\int_{(0, t]} A(s-) \mathrm{d} X(s)-\mathbb{E} X(1) \int_{0}^{t} A(s) \mathrm{d} s}{t} \rightarrow 0 \quad \text { a.s. }
$$

Proof. Assume that $|A(t)| \leq B<\infty$. Set, for $M>0$,

$$
\begin{aligned}
X_{M}(t) & =\sum_{0<s \leq t} \Delta X(s) \mathbf{1}_{\{\Delta X(s)>M\}}, \\
X_{-M}(t) & =\sum_{0<s \leq t} \Delta X(s) \mathbf{1}_{\{\Delta X(s)<-M\}}, \\
X_{0}(t) & =X(t)-X_{M}(t)-X_{-M}(t) .
\end{aligned}
$$

Also, define $\xi_{i}=\mathbb{E} X_{i}(1)$ for $i=M,-M, 0$. Then $X_{M}, X_{-M}$, and $X_{0}$ are independent Lévy processes. $X_{M}$ is nondecreasing and $X_{-M}$ is nonincreasing. Now,

$$
\left|\frac{1}{t} \int_{(0, t]} A(s-) \mathrm{d} X_{M}(s)\right| \leq B \frac{X_{M}(t)}{t},
$$

and by the strong law of large numbers for Lévy processes we have, a.s.,

$$
\limsup _{t \rightarrow \infty}\left|\frac{1}{t} \int_{(0, t]} A(s-) \mathrm{d} X_{M}(s)\right| \leq B \xi_{M}=B \int_{(M, \infty)} x v(\mathrm{~d} x) .
$$

Clearly, we also have

$$
\left|\frac{1}{t} \int_{0}^{t} A(s) \mathrm{d} s\right| \leq B
$$

and, thus,

$$
\limsup _{t \rightarrow \infty}\left|\frac{\int_{(0, t]} A(s-) \mathrm{d} X_{M}(s)-\xi_{M} \int_{0}^{t} A(s) \mathrm{d} s}{t}\right| \leq 2 B \int_{(M, \infty)} x v(\mathrm{~d} x) .
$$

Similarly,

$$
\limsup _{t \rightarrow \infty}\left|\frac{\int_{(0, t]} A(s-) \mathrm{d} X_{-M}(s)-\xi_{-M} \int_{0}^{t} A(s) \mathrm{d} s}{t}\right| \leq 2 B \int_{(-\infty,-M)}|x| v(\mathrm{~d} x) .
$$


Next, we observe that the martingale $M_{0}(t)=X_{0}(t)-\xi_{0} t$ is a Lévy process with bounded jumps and, thus, its quadratic variation is a nondecreasing Lévy process with bounded jumps which can also be compensated by a linear function to create a martingale (as in Lemma 3). Thus, as in the proof of Theorem 2, this implies that

$$
\frac{\int_{(0, t]} A(s-) \mathrm{d} X_{0}(s)-\xi_{0} \int_{0}^{t} A(s) \mathrm{d} s}{t} \rightarrow 0 \quad \text { a.s. }
$$

(and also in $L^{2}$, but this is not needed here). To conclude, defining $\xi=\xi_{0}+\xi_{M}+\xi_{-M}=\mathbb{E} X(1)$, we now clearly have, a.s.,

$$
\limsup _{t \rightarrow \infty}\left|\frac{\int_{(0, t]} A(s-) \mathrm{d} X(s)-\xi \int_{0}^{t} A(s) \mathrm{d} s}{t}\right| \leq 2 B \int_{(-\infty,-M) \cup(M, \infty)}|x| v(\mathrm{~d} x),
$$

and letting $M \rightarrow \infty$, recalling that $\int_{|x|>1}|x| v(\mathrm{~d} x)<\infty$, the proof is complete.

Remark 2. (Relation with PASTA.) We note that if $\mathbb{E}|X(1)|<\infty$ and $\mathbb{E} X(1) \neq 0$ then, since $X(t) / t \rightarrow \mathbb{E} X(1)$ a.s., (5) is equivalent to

$$
\frac{1}{X(t)} \int_{(0, t]} A(s-) \mathrm{d} X(s)-\frac{1}{t} \int_{0}^{t} A(s) \mathrm{d} s \rightarrow 0,
$$

and, thus, $(X(t))^{-1} \int_{(0, t]} A(s-) \mathrm{d} X(s)$ converges a.s. if and only if $t^{-1} \int_{0}^{t} A(s) \mathrm{d} s$ does, and the limits coincide. When $X$ is a Poisson process, this is no less than an equivalent statement of the famous and often cited PASTA (Poisson arrivals see time averages) property. See [12] for a martingale approach in a (nonexplosive) point process setting.

An immediate corollary of Lemma 4 is the following, where $v_{k}$ is the (marginal) Lévy measure associated with $X_{k}$.

Corollary 3. (Strong law for $\tilde{X}$.) Assume that $\int_{|x|>1}|x| v_{k}(\mathrm{~d} x)<\infty$ (equivalently, $\mathbb{E}\left|X_{k}(1)\right|<$

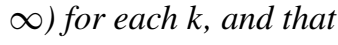

$$
\frac{1}{t} \int_{0}^{t} I_{k}(s) \mathrm{d} s \rightarrow \beta_{k}
$$

a.s. as $t \rightarrow \infty$. Then, a.s.,

$$
\xi=\lim _{t \rightarrow \infty} \frac{\tilde{X}(t)}{t}=\sum_{k=1}^{K} \beta_{k} \mathbb{E} X_{k}(1) .
$$

Thus, we can summarize with the following.

Theorem 3. Assume that, for each $k$, the $X_{k}$ have no negative jumps, $\int_{x>1} x v_{k}(\mathrm{~d} x)<\infty$ (equivalently, $\left.\mathbb{E} X_{k}(1)<\infty\right)$, and that the $I_{k}$ are nonnegative with

$$
\frac{1}{t} \int_{0}^{t} I_{k}(s) \mathrm{d} s \rightarrow \beta_{k}
$$

a.s. as $t \rightarrow \infty$. Then, a.s.,

$$
\frac{1}{t} \int_{0}^{t} \varphi(I(s)) \mathrm{e}^{-Z(s)} \mathrm{d} s \rightarrow-\left(\sum_{k=1}^{K} \beta_{k} \mathbb{E} X_{k}(1)\right)^{-}
$$


Note that, when $K=1$ and $I_{1}(t)=\alpha$ for all $t$, we have $\beta_{1}=\alpha$ and $\xi=\mathbb{E} X_{1}(1)=-\varphi^{\prime}(0)$. This immediately implies that if $\varphi^{\prime}(0)>0$ then

$$
\frac{1}{t} \int_{0}^{t} \mathrm{e}^{-Z(s)} \mathrm{d} s \rightarrow \frac{\alpha \varphi^{\prime}(0)}{\varphi(\alpha)} \quad \text { as } t \rightarrow \infty .
$$

When $\varphi^{\prime}(0)<0$ or $\varphi^{\prime}(0)=0$ but $X_{1}$ is not identically 0 (so that $\varphi(\alpha)>0$ for each $\alpha>0$ ), then the limit is 0 . When $\varphi^{\prime}(0)>0$, this limit is the well-known generalized Pollaczek-Khinchine formula. We also observe that $\varphi^{\prime}(0)<0$ is the transient case and $\varphi^{\prime}(0)=0$ but $X_{1}$ is not identically 0 is the null recurrent case, so that neither is really a big surprise, but it is nice to see that it also follows directly from the above.

\section{References}

[1] Applebaum, D. (2004). Lévy Processes and Stochastic Calculus. Cambridge University Press.

[2] Asmussen, S. (2003). Applied Probability and Queues, 2nd edn. Springer, New York.

[3] Asmussen, S. and Kella, O. (2000). A multi-dimensional martingale for Markov additive processes and its applications. Adv. Appl. Prob. 32, 376-393.

[4] Asmussen, S. And Kella, O. (2001). On optional stopping of some exponential martingales for Lévy processes with or without reflection. Stoch. Process. Appl. 91, 47-55.

[5] Boxma, O. J., Ivanovs, J., Kosiński, K. M. And MandJes, M. R. H. (2011). Lévy-driven polling systems and continuous-state branching processes. Stoch. Systems 1, 411-436.

[6] Kella, O. (1998). An exhaustive Lévy storage process with intermittent output. Commun. Statist. Stoch. Models 14, 979-992.

[7] Kella, O. (2006). Reflecting thoughts. Statist. Prob. Lett. 76, 1808-1811.

[8] Kella, O. And Whitt, W. (1991). Queues with server vacations and Lévy processes with secondary jump input. Ann. Appl. Prob. 1, 104-117.

[9] Kella, O. ANd Whitt, W. (1992). Useful martingales for stochastic storage processes with Lévy input. J. Appl. Prob. 29, 396-403.

[10] Kella, O. ANd Whitt, W. (1996). Stability and structural properties of stochastic storage networks. J. Appl. Prob. 33, 1169-1180.

[11] Kyprianou, A. E. (2006). Introductory Lectures on Fluctuations of Lévy Processes with Applications. Springer, Berlin.

[12] Melamed, B. And Whitt, W. (1990). On arrivals that see time averages: a martingale approach. J. Appl. Prob. 27, 376-384.

[13] NGuYEn-NGoc, L. And Yor, M. (2005). Some martingales associated to reflected Lévy processes. In Séminaire de Probabilités XXXVIII (Lecture Notes Math. 1857), Springer, Berlin, pp. 42-69.

[14] Protter, P. E. (2005). Stochastic Integration and Differential Equations, 2nd edn. Springer, Berlin.

[15] RüDIGER, B. (2004). Stochastic integration with respect to compensated Poisson random measures on separable Banach spaces. Stoch. Stoch. Reports 76, 213-242. 